\title{
DGN-Summer School 2019
}

Die sechste DGN-Summer School findet in der Zeit vom 29. bis 31. August 2019 an der Charité in Berlin zum Thema „Translational Research in Molecular Imaging and Radionuclide Therapy“ statt. Die wissenschaftliche Leitung liegt erneut bei Prof. Dr. Winfried Brenner.

Die DGN-Summer School ist ein internationales englischsprachiges Forum, das sich an Studierende, Doktoranden sowie junge Nachwuchswissenschaftler in den ersten Jahren ihrer Weiterbildung in den Bereichen der (Nuklear-)Medizin, Physik und (Radio-) Chemie richtet. Ziel ist es, den wissenschaftlichen Nachwuchs für die Nuklearmedizin und die molekulare Bildgebung zu begeistern, in Forschungsaktivitäten zu integrieren und langfristig in die translationalen Bereiche des Fachgebiets einzubinden.

Maximal 30 Teilnehmer werden im Rahmen der dreitägigen DGN-Summer School in Vorträgen, Falldemonstrationen sowie Hands-
on-Veranstaltungen verschiedene Themen wissenschaftlich fundiert und praxisnah aktiv kennenlernen und gemeinsam mit den Dozenten intensiv diskutieren können. Als Referenten werden hochkarätige nationale wie auch internationale Experten des jeweiligen Themenbereichs eingeladen. Gemeinsame Abendveranstaltungen runden das Programm ab und ermöglichen auch außerhalb der Veranstaltungen einen regen Austausch zwischen Teilnehmern und Tutoren.

\section{Teilnahme}

Die Auswahl der Teilnehmer für die DGNSummer School erfolgt auf Grundlage der einzureichenden Bewerbungsunterlagen:

- Lebenslauf,

- Motivationsschreiben,

- Unterstützungsschreiben des Leiters Ihrer Einrichtung, an der Sie beschäftigt sind.

Bitte senden Sie Ihre Bewerbung - in Deutsch oder Englisch - per Mail bis zum
31. Mai 2019 an die DGN-Geschäftsstelle. Später eintreffende Bewerbungen können im Auswahlverfahren leider nicht mehr berücksichtigt werden. Erfolgreiche Bewerber erhalten ein Stipendium, das Übernachtungs- und Verpflegungskosten sowie die Kurskosten abdeckt.

Allen interessierten Nachwuchswissenschaftlern und an der Unterstützung der Summer School interessierten Unternehmen steht die DGN-Geschäftsstelle jederzeit gerne per E-Mail unter office@nuklearmedizin.de oder telefonisch unter 0551 48857-401 für weitere Auskünfte zur Verfügung. 